\title{
Gamma-Ray Bursts as Cosmological Probes
}

\author{
Tomonori Totani \\ Department of Astronomy, Kyoto University, \\ Sakyo-ku, Kyoto 606-8502, Japan \\ email: totani@kusastro.kyoto-u.ac.jp
}

\begin{abstract}
The status and prospects for gamma-ray bursts (GRBs) as cosmological probes are reviewed. Long duration GRBs can potentially be used as an indicator of star formation rate (SFR), though GRB rate might be systematically different from SFR, by the effect of e.g. metallicity. There are several papers claiming that the cosmic GRB rate history is different from that of SFR in the sense that GRB rate is relatively higher than SFR at higher redshifts, which may be explained by the metallicity effect. However, considering the large uncertainties about the efficiency of GRB afterglow detection and redshift determination, it would be conservative to state that the observed GRB rate is roughly consistent with the star formation history. GRBs can also be used as a unique and powerful tool to reveal the reionization history. However, there is practically no progress in this direction since the first GRB-based useful constraint on reionization in 2005 (GRB 050904). The bottleneck now is the insufficient sensitivity of nearinfrared spectroscopy, even with $8 \mathrm{~m}$ class telescopes. The planned $30 \mathrm{~m}$ class telescopes will bring the next breakthruough. Finally, GRBs can potentially be used as a standard candle to study cosmology by a geometrical test. However, there are still many steps for GRBs to overcome before it produces a result that has strong impact on the cosmology community in the precision cosmology era.
\end{abstract}

Keywords. cosmology: miscellaneous, gamma rays: bursts

\section{Introduction}

Although more than 30 years have passed since the discovery of gamma-ray bursts (GRBs) [see, e.g., Mészáros (2002), Piran (2004) for recent reviews], it was rather recent that GRBs became widely recognized as a unique tool of cosmological studies and exploring the early universe. It was just before GRBs were proved to have the cosmological origin in 1997 that the first attempt to study the cosmic star formation history by using GRBs was made (Totani 1997, Wijers et al. 1998). The potential use of GRBs as a probe of the reionization history of intergalactic medium (IGM) was also pointed out (Miralda-Escudé 1998) soon after GRBs were proven to be cosmological sources. At that time, it was thought that GRBs can probe the universe at most up to modest redshifts of $z \sim 3$ by instruments available at that time or in the near future. Such notion was, however, soon discarded by the discoveries of extremely luminous GRBs like GRB 971214 and 990123, which could be detected even at redshifts beyond 10. Then it did not take long time before astrophysicists started to discuss GRBs as a promising lighthouse to study the extremely high redshift universe (e.g., Lamb \& Reichart 2000), potentially giving cosmologically important information including the population III star formation and the reionization history.

During 2000-2004, satellites such as the BeppoSAX and the HETE-2 continued to discover more and more GRBs, and there was important progress including the establishment of the firm connection between long duration GRBs and energetic supernovae. However, the distance of GRBs did not extend to very high redshift, with the highest record of $z=4.5$ (Andersen et al. 2000). The launch of the Swift satellite in 2004 then 
allowed the GRB community to search for fainter and more distant GRBs with improved detection rate. The first breakthrough was brought by GRB 050904 at $z=6.3$ (Kawai et al. 2006; Totani et al. 2006), and it kept its record more than two years until broken by GRB 080913 at $z=6.7$ (Greiner et al. 2009). Furthermore, in 2009 GRB 090423 was discovered at $z=8.3$ (Salvaterra et al. 2009; Tanvir et al. 2009), placing GRBs at the top rank in the list of the most distant astronomical source populations, far beyond the most distant galaxy at $z=6.96$.

Now fifteen years have already passed since gamma-ray bursts were revealed to be objects at cosmological distances. Since then, various possibilities of using GRBs as cosmological probes have been discussed, and applied to derive useful cosmological information. (Here, I use the term "cosmology" in a relatively wide context including galaxy formation, not only more cosmological issues such as cosmological parameters, dark energy, etc.)

Here I review the status and prospects of the three important applications of GRBs as cosmological probes: GRBs as an indicator of star formation rate and cosmic star formation history, GRBs as a probe of cosmic reionization, and GRBs as a standard candle to study cosmic expansion by a geometrical test.

\section{GRBs as a Star Formation Indicator}

The idea of using GRBs as a SFR indicator is simple; since GRBs are related to the death of massive stars, the time from star formation to GRB events are much shorter than the cosmological time scale, and hence GRB rate is expected to trace SFR. Compared with various SF indicators of galaxies, GRBs have advantages of (1) being free from extinction by dust, (2) reaching to very high redshifts, and (3) free from the detection limit about host galaxy luminosity. Note that this is applicable only for long duration GRBs, for which the association with massive supernovae has been established. Short duration GRBs are known to occur also in galaxies having old stellar populations, and hence it cannot be a direct SFR indicator (see below).

There are a number of papers that tried to reconstruct GRB rate history from the observed GRB redshift distribution, and compare it with the cosmic star formation history based on SFR estimates of galaxies at various redshifts. Many papers found and claimed that GRB rate evolution is systematically different from that of SFR, in the sense that GRB rate becomes relatively higher than SFR with increasing redshifts (see Campisi et al. 2010; Qin et al. 2010; Wanderman et al. 2010 for recent results and references therein for earlier ones). This may indicate some physical effects about the condition for GRB events to occur. The most popular scenario is the metallicity effect; theoretically GRBs are expected to preferentially occur in low metallicity environment, because low metallicity is useful to suppress stellar mass loss and keep large total stellar mass and angular momentum. If the low metallicity preference of GRBs is correct, indeed we expect higher GRB rate than SFR with increasing redshift.

However, this result might be a result of selection effects. The difficulty to reconstruct a correct GRB rate history from observation is the treatment of GRB detection efficiency. This efficiency includes not only that for detecting the prompt gamma-ray emission, but also those for afterglow detection and redshift determination. There are many observational steps to determine redshift for a GRB, and the selection effects must be complicated.

In fact, recent studies using latest, relatively homogeneous samples indicate that the major origin of "dark" GRBs is large extinction of optical afterglow. This means that the past GRB sample might have been biased to less dusty, low mass and low metallicity host galaxies. Using the latest sample taking into account this effect, Elliott et al. (2012) 
found that GRB rate history is consistent with the star formation history. [But see also Salvaterra et al. (2012) who found an opposite result using another complete sample taking into account detection efficiency.] This demonstrates that the importance and difficulty of correctly incorporating selection effects and detection efficiency in the rate studies of GRBs.

As for short-duration GRBs, currently the sample size of GRBs with known redshifts is still small, making it difficult to derive strong constraint on the rate evolution over the cosmic time. However, more detailed studies will become possible in the near future. What is important for rate studies for short GRBs is the delay time distribution (DTD), since we know that short GRBs occur with a large delay time from star formation. A hint is coming from the rate studies for type Ia supernovae (SNe Ia). Recent studies indicate that DTD of SNe Ia is described as $\propto t^{-1}$ (Totani et al. 2008; Maoz \& Mannucci 2012). It is interesting to note that this form of DTD is a general prediction of souces whose delay time is determined by gravitational wave radiation in binaries (Totani 1997; Totani et al. 2008). Therefore the $t^{-1}$ DTD of SNe Ia can easily be explained if the progenitor of SNe Ia is double-degenerate (two white dwarfs) binaries, though it does not simply exclude another popular progenitor scenario of SNe Ia (single-degenerate binaries). The double neutron star binaries or NS-BH binaries, which are discussed as a popular scenario of short GRBs, also predict this form of DTD, and future short GRB rate studies should examine the consistency between short GRB rate evolution and SFR evolution under this type of DTD.

\section{GRBs as a Probe of Reionization}

The famous Gunn-Peterson (GP) test tells us that the IGM is highly ionized at $z \lesssim 5$, while the observations of the cosmic microwave background radiation (CMB) indicates that the universe became neutral at the recombination epoch of $z \sim 1100$. The reionization of the IGM is believed to have occurred during $z \sim 6-20$ by the first stars and/or quasars, and the precise epoch and nature of the reionization is one of the central topics in the modern cosmology (see e.g. Loeb \& Barkana 2001 for recent reviews). The dramatic increase of the optical depth of the Ly $\alpha$ forest with increasing redshift at $z \gtrsim 5.2$ and the subsequent discovery of broad and black troughs of Ly $\alpha$ absorption (the GP troughs) in the spectra of $z \gtrsim 6$ quasars indicate that we are beginning to probe the epoch of reionization. On the other hand, the polarization observation of the CMB by the Wilkinson Microwave Anisotropy Probe (WMAP) indicates a much higher redshift of reionization, $z \sim 10$. Some theorists have argued that the hydrogen in the IGM could have been reionized twice.

Because the cross section of the Ly $\alpha$ resonance absorption is so large, the light blueward of the Ly $\alpha$ wavelength at the source is completely attenuated if the IGM neutral fraction $x_{\mathrm{HI}} \equiv n_{\mathrm{HI}} / n_{\mathrm{H}}$ is larger than $\sim 10^{-3}$, and hence the Ly $\alpha$ trough of $z \sim 6$ quasars gives a constraint of only $x_{\mathrm{HI}} \gtrsim 10^{-3}$. The cross section becomes much smaller for longer wavelength photons than the Ly $\alpha$ resonance, and the spectral shape of the red damping wing of the GP trough can potentially be used to measure $x_{\mathrm{HI}}$ more precisely (MiraldaEscudé 1998). However, applying this method to quasars is problematic because of the uncertainties in the original unabsorbed quasar spectra and the proximity effect, i.e., the ionization of surrounding IGM by strong ionizing flux from quasars. Therefore, though some authors suggested $x_{\mathrm{HI}} \gtrsim 0.1$ using $z \sim 6$ quasar spectra, these estimates are generally model dependent.

The Ly $\alpha$ line emission is seriously attenuated if it is embedded in the neutral IGM, and hence the Ly $\alpha$ line emissivity of galaxies at $z \gtrsim 6$ is another probe of the reionization. 
Therefore the detection of Ly $\alpha$ emission from many galaxies at $z \gtrsim 6$ may indicate that the universe had already been largely ionized at that time. However it should not be naively interpreted as implying small $x_{\mathrm{HI}}$, since these Ly $\alpha$ emitters (LAEs) are selected by strong Ly $\alpha$ emission and hence they may be biased to those in ionized bubbles created by themselves or clusters of undetected sources. On the other hand, the Lyman-break galaxies (LBGs) selected by broad-band colors are free from the selection bias about Ly $\alpha$ emission, but the statistical nature of Ly $\alpha$ emission from LBGs at $z \gtrsim 5$ is not yet well understood, compared with those at $z \sim 3$. Furthermore, the Ly $\alpha$ line emission from lower-redshift starburst galaxies is often redshifted with respect to the systemic velocity of galaxies, and such a relative redshift will lead to a higher detectability of LAEs at $z \gtrsim 6$, indicating a possible systematic uncertainty in the reionization study by Ly $\alpha$ emission.

GRBs have a few advantages as a probe of the cosmic reionization, compared with quasars or LAEs/LBGs. GRB afterglows are much brighter than LAEs/LBGs and comparable to or even brighter than quasars if they are observed quickly enough after the explosion. The Ly $\alpha$ or ultraviolet luminosity of host galaxies is irrelevant to the detectability of GRBs, and hence GRBs can probe less biased regions in the early universe, while quasars and bright LAEs/LBGs are likely biased to regions of rapid structure formation with strong clustering. In most cases it is expected that the IGM ionization state around GRB host galaxies had not yet been altered by strong ionizing flux from quasars. Finally, the spectrum of GRB afterglows has a much simpler power-law shape than complicated lines and continuum of quasars and LAEs/LBGs, and hence model uncertainty can greatly be reduced. Especially, a detailed fitting analysis of the damping wing of the GP trough in an afterglow spectrum may lead to a precise determination of $x_{\mathrm{HI}}$.

In the following of this section, I summarize the knowledge we obtained from GRB 050904 (the only useful constraint on reionization by GRBs), and discuss the present status and future prospects.

\subsection{Lessons from GRB 050904}

The GRB 050904 was discovered by the Swift on 2005 September 4 at 01:51:44 UT, and follow-up photometric observations of the afterglow found a strong spectral break between optical and near-infrared (NIR) bands, indicating a very high redshift of $z \sim$ 6. This suggestion was confirmed by the subsequent spectroscopic observation by the Subaru Telescope, which found metal absorption lines at $z=6.295$ and the corresponding Lyman break and red damping wing (Kawai et al. 2006). This opened a new era of GRB observations at redshifts that are close to the cosmic reionization and comparable to those of the most distant galaxies and quasars.

The detection of red damping wing by neutral hydrogen could have a significant impact on reionization if it is caused by neutral hydrogen in intergalactic medium. However, it could also be caused by neutral hydrogen in the host galaxy of the GRB. In fact, many damped Ly $\alpha$ systems (DLAs) have been observed in GRB afterglow spectra, like those in quasar spectra. The key issue is how to discriminate between the two possibilities.

Since the damping wing by intergalactic hydrogen is a superposition of hydrogen at many different redshifts, the shape and location of the wing are slightly different from those of DLA in the host galaxy. Although the shape difference is difficult to discriminate with the quality of GRB 050904 spectrum, the location of IGM wing is shifted to blueward compared with DLA wings and they can be discriminated if the host redshift is precisely known. Because of the high quality of the afterglow spectrum, several absorption lines were detected, giving strong constraints on the exact redshift of the host galaxy. There are several physical effects that could produce systematic shift of observed absorption 
lines from the true systemic redshift of the host, as discussed in Totani et al. (2006), and these must be carefully taken into account when one derives constraint on reionization. Another feature useful to discriminate between IGM and DLA is Ly $\beta$ feature, because it also gives a clear constraint on the host redshift.

By using the absorption line information and Ly $\beta$ feature, Totani et al. (2006) showed that the damping wing is caused by DLA, and absence of evidence for IGM wing gives an upper limit on neutral hydrogen fraction at $z=6.3$. They derived $x_{\mathrm{HI}}<0.17$ and 0.60 at 68 and $95 \%$ C.L., respectively. This is complementary to the lower bounds on $x_{\mathrm{HI}}$ from quasar GP tests, proving that GRBs are a powerful and unique tool to study reionization.

\subsection{GRB 080913 and $G R B 090423$}

In contrast to GRB 050904, no useful constraints on reionization was derived from afterglow spectra of GRB 080913 and 090423. The main reason is that the signal-to-noise is much lower than that of GRB 050904, even though these two bursts were observed at much shorter time after the burst compared with the case of GRB 050904 (3 days). This is simply because the absolute luminosity of GRB 050904 afterglow was exceptionally bright. The absolute luminosities of GRB afterglows are widely distributed over more than two orders of magnitude. Compared with other past afterglows after scaled to the same redshift and band filter, GRB 050904 optical afterglow was one of the brightest among all the afterglows observed in the past. On the other hand, GRB 080913 and 090423 had afterglow luminosities that are close to the average. The situation is even more severe for GRB 090423, because its spectrum at $z=8.3$ is only accessible with near-infrared spectrographs, whose sensitivity is much worse than optical spectrographs used to marginally detect Lyman breaks of GRB 050904 and 080913 spectra. Both the spectra of GRBs 080913 and 090423 were taken by VLT, and this indicates that even the state-of-art 8m-class telescopes are not sufficient to study reionization by using GRBs of normal luminosities.

\subsection{Future Prospects}

Since the currently available large telescopes do not have enough power, we need more powerful instruments in the future to probe reionization by GRBs. An obvious solution is planned 30m-class telescopes and the James Webb Space Telescope. These facilities would have more than 100 times better spectroscopic sensitivities against point sources for Lyman break at $z \gtrsim 7$ than current $8 \mathrm{~m}$-class telescopes, and it would be possible to perform detailed studies of afterglow spectra including absorption lines, even for normalluminosity GRB afterglows. However, the ultimate limitation for the reionization study by GRBs would come from their event rate. The statistics of very high- $z$ GRBs searched by Swift (only 3 detected GRBs at $z>6$ in five years) indicates that the fraction of very high redshift GRBs is not very high. Therefore, it is important to launch GRB satellites during the era of $30 \mathrm{~m}$ telescopes and JWST, which have high sensitivity to trigger faint gamma-ray flux, and a wide field of view to increase the GRB detection rate. There are several proposals for such missions, e.g., EXIST. It should also be noted that, even if the expected event rate is not very high, only a few events of very high- $z$ GRBs would have strong impact on reionization study, because GRBs are the only one source population that can be used to precisely measure $x_{\mathrm{HI}}$ by damping wing. 


\section{GRBs as a Standard Candle}

There are several types of correlations between GRB absolute luminosity (or total emitted gamma-ray energy) and spectral features, e.g., spectral peak energy $\left(E_{\text {peak }}\right)$ versus isotropic energy $\left(E_{\text {iso }}\right)$ (Amati et al. 2002), or $E_{\text {peak }}$ versus isotropic luminosity $\left(L_{\text {iso }}\right)$ (Yonetoku et al. 2004). These correlation can be used to correct and calibrate GRB luminosities to a standard candle, in a similar way to that of the period-luminosity relation of Cepheid variables or the peak luminosity versus stretch relation for type Ia supernovae. A number of papers already tried to use gamma-ray bursts as a standard candle and derive some constraints on cosmological parameters. Advantages of GRBs compared with the other cosmological standard candle, SNe Ia, are (1) being free from the extinction in host galaxies and (2) reach to much higher redshifts.

However, unfortunately, results on cosmological parameters by GRBs have not yet had strong impact on the cosmological community. The main reason for this is the still very large systematic uncertainty of GRBs as a standard candle. The dispersion around the mean relations (e.g., a factor of several for $E_{\text {peak }}-E_{\text {iso }}$ ) is much larger than that of SNe Ia $(\sim 10-20 \%)$. In the cosmology community, many people think that even SNe Ia is now limited by systematic uncertainties, and there is a huge effort to standardize $\mathrm{SNe}$ Ia with higher accuracy to derive reliable constraints on the dark energy parameter such as $w \equiv p / \rho$ and its time derivative.

An obvious concern about the correlations of GRB quantities used to standardize GRBs is the selection effect. Most of the proposed relations tell us that the total energy or luminosity of GRBs increases with increasing characteristic photon energy of GRB spectra. It should be noted that this is something expected by a selection effect about a fixed photon energy range of GRB detectors. When GRBs are preferentially detected at a fixed observed photon energy range, the rest-frame spectral peak energy would increase with increasing redshifts, and at the same time, the absolute luminosity will also increase by the detection flux limit of the detector.

It is probably impossible to explain all the observed GRB correlations simply by the selection effect, and the astrophysical origin of the observed correlation is a quite interesting topic. However, such a selection effect is certainly significant when GRBs are applied to precision cosmology. To conclude, although GRBs may potentially be a standard candle that is useful for cosmological tests, there are still many steps for GRBs to overcome before producing a cosmological result having a strong impact on the cosmological community.

\section{References}

Amati, L., Frontera, F., Tavani, M., et al. 2002, A\&\&A, 390, 81

Andersen, M. I., et al. 2000, A\& A, 364, L54

Campisi, M. A., Li, L.-X., \& Jakobsson, P. 2010, MNRAS, 407, 1972

Elliott, J., Greiner, J., Khochfar, S., et al. 2012, A\&AA, 539, A113

Greiner, J., et al. 2009, ApJ, 693, 1610

Kawai, N., et al. 2006, Nature440, 184

Lamb, D. Q. \& Reichart, D. E. 2000, ApJ, 536, 1

Loeb, A. \& Barkana, R. 2001, ARA\&A, 39, 19

Maoz, D. \& Mannucci, F. 2011, arXiv:1111.4492

Mészáros, P. 2002, ARA\&A, 40, 137

Miralda-Escudé, J. 1998, ApJ, 501, 15

Piran, T. 2004, Rev. Mod. Phys. 76, 1143

Qin, S.-F., Liang, E.-W., Lu, R.-J., Wei, J.-Y., \& Zhang, S.-N. 2010, MNRAS, 406, 558 
Salvaterra, R. et al. 2009, Nature, 461, 1258

Salvaterra, R. et al. 2012, ApJ, 749, 68

Tanvir, N. R., et al. 2009, Nature, 461, 1254

Totani, T. 1997, ApJ, 486, L71

Totani, T., et al. 2006, PASJ, 58, 485

Totani, T., Morokuma, T., Oda, T., Doi, M., \& Yasuda, N. 2008, PASJ, 60, 1327

Wanderman, D. \& Piran, T. 2010, MNRAS, 406, 1944

Wijers, R. A. M. J., Bloom, J. S., Bagla, J. S., \& Natarajan, P. 1998, MNRAS, 294, L13

Yonetoku, D., Murakami, T., Nakamura, T., et al. 2004, ApJ, 609, 935

\section{Discussion}

AmATi: Concerning the use of GRBs for cosmological parameters, there is evidence that the $E_{\text {peak }}-E_{\text {iso }}\left(L_{\text {iso }}\right)$ correlations are not significantly affected by selection effects.

TOTANi: It depends on the required accuracy as a standard candle whether the selection effect is significant or not. Currently, the scatter along the mean relation of GRBs is still much larger than SNe Ia, and only weak constraints on $\Omega_{M}$ and $\Omega_{\Lambda}$ have been derived. In such analysis, the selection effect may not be significant. But I guess it should be significant if GRBs are used to derive much more precise cosmological constraints, such as $w$. 